\title{
DESENVOLVIMENTO DE PLANILHA ELETRÔNICA PARA O MANEJO DA IRRIGAÇÃo
}

Mairton Gomes da Silva ${ }^{1}$, Francisco Dirceu Duarte Arraes $^{2}$, Eder Ramon Feitoza Ledo ${ }^{3}$, Dijauma Honório Nogueira ${ }^{4}$

\section{RESUMO}

No trabalho é apresentado o desenvolvimento de planilhas eletrônicas para o controle do manejo da irrigação. As planilhas foram desenvolvidas em linguagem de programação Visual Basic for Applications (VBA). As planilhas foram testadas com a simulação da determinação das necessidades hídricas da cultura do melão, obtendo-se dados de evaporação e coeficiente do tanque Classe "A" para estimativa da evapotranspiração de referência (ETo ) e coeficientes

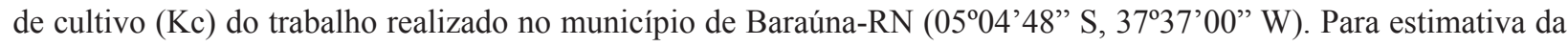
$\mathrm{ET}_{\mathrm{o}}$ pelos demais métodos disponíveis nas planilhas, foram obtidos dados diários de temperatura máxima e mínima do ar, umidade relativa do ar, velocidade do vento e número de horas de insolação da estação convencional de MossoróRN, compreendendo o período de dezembro de 2005 a março de 2006. A demanda hídrica do melão com estimativas de ET ${ }_{0}$ pelo tanque Classe "A" simulada nas planilhas foi próxima dos valores obtidos no experimento. O conjunto de planilhas mostrou-se prático e rápido na execução dos cálculos envolvidos na determinação das necessidades hídricas da cultura.

Palavras-chave: evapotranspiração da cultura, irrigação, elementos climáticos

\section{ABSTRACT \\ DEVELOPMENT OF AN ELECTRONIC SPREADSHEET FOR IRRIGATION MANAGEMENT}

This study presented the development of electronic spreadsheets for the control of irrigation management. Spreadsheets were developed in the programming language Visual Basic for Applications (VBA). These spreadsheets were tested by simulations for determining the water requirements of the melon crop, yielding evaporation data and the Class " $\mathrm{A}$ " pan coefficient for estimating reference evapotranspiration $\left(\mathrm{ET}_{\mathrm{o}}\right)$ and crop coefficients $(\mathrm{Kc})$ for the study conducted in the municipality of Baraúna-RN $\left(05^{\circ} 04^{\prime} 48^{\prime \prime} \mathrm{S}, 37^{\circ} 37^{\prime} 00^{\prime \prime} \mathrm{W}\right)$. To estimate the $\mathrm{ET}_{\mathrm{o}}$ by the methods available in the spreadsheets, daily data of the maximum and minimum temperature of the air, relative humidity of the air, wind speed and number of hours of sunshine were obtained from the Mossoró-RN conventional station, comprising the period from December 2005 to March 2006. The water demand for melon with estimates of ET by Class "A" pan simulated in the spreadsheets was similar to obtained experimental values. The set of spreadsheets showed to be practical and fast in the execution of calculations involved in the determination of water requirements of the crop.

Keywords: crop evapotranspiration, irrigation, climatic elements

\section{Recebido para publicação em 03/08/2012. Aprovado em 28/02/2013.}

1 - Tecnólogo em Irrigação e Drenagem, Mestrando em Eng. Agrícola na UFRB/Cruz das Almas - BA, e-mail: mairtong@hotmail.com

2 - Tecnólogo em Irrigação e Drenagem, Doutorando em Eng. de Sistemas Agrícolas na ESALQ/USP, Prof. Substituto do IFCE/ Campus Iguatu, e-mail: dirceuarraes@gmail.com

3 - Tecnólogo em Irrigação e Drenagem, Mestrando em Eng. Agrícola na UFC/Fortaleza - CE, e-mail: eder_ramon@hotmail.com 4 - Prof. do IFCE/Campus Iguatu, e-mail: dijaumahonorio@ifce.edu.br 


\section{INTRODUÇ̃̃O}

$\mathrm{Na}$ maioria das áreas irrigadas é comum observar ausência de manejo racional da água, geralmente resultando em aplicação excessiva, com desperdício de água e energia, além da ocorrência de problemas ambientais ou em deficiência hídrica para as plantas, com baixa produtividade e prejuízos econômicos ao produtor (OLIVEIRA et al., 2011).

O conhecimento do consumo hídrico das culturas constitui-se numa informação preciosa no manejo da água, principalmente no momento em que ocorre forte conscientização popular do uso racional dos recursos hídricos (SILVA et al., 2005).

$\mathrm{Na}$ estimativa da exigência hídrica pelas plantas, podem ser adotadas metodologias distintas ou a integração delas, ou seja, pela demanda atmosférica, pelo monitoramento da umidade do solo e por meio de medidas diretas do fluxo de água na planta. Essas metodologias objetivam apresentar alternativas do manejo da água nas culturas irrigadas em qualquer estádio fenológico da planta (BARRETO et al., 2004).

O conhecimento da evapotranspiração de culturas (ETc) é fundamental para que se tenha um manejo adequado da irrigação, principalmente em regiões como o semiárido nordestino, em que a escassez e a irregularidade pluviométrica são fatores limitante da produção agrícola (OLIVEIRA et al.; 2010).

A determinação das necessidades hídricas das culturas é usualmente estimada com base nos valores da evapotranspiração de referência $\left(\mathrm{ET}_{\mathrm{o}}\right)$, associado a um coeficiente de cultivo (BUSATO; BUSATO, 2011; OLIVEIRA et al.; 2011). Em razão dos diversos métodos existentes para estimativa da $\mathrm{ET}_{\text {o }}$, a escolha do mais adequado depende da disponibilidade de dados meteorológicos, do nível de precisão exigido, da finalidade (manejo da irrigação ou pesquisa) e do custo de aquisição dos equipamentos (OLIVEIRA et al.; 2011).

Diante do grande avanço da informática, ferramentas computacionais foram desenvolvidas para o controle no manejo da irrigação. Citandose as planilhas desenvolvidas para a programação de irrigação de culturas anuais (ALBUQUERQUE; ANDRADE, 2001); para o manejo da irrigação na produção integrada de caju (MIRANDA, 2005); para o planejamento de calendários de irrigação (PINTO et al., 2007); para o manejo da irrigação em pequenas propriedades (CONCEIÇÃO, 2010); para o manejo da irrigação na cultura da mangueira (PAULA et al., 2011); para o controle da irrigação na cultura da videira (NASCIMENTO et al., 2012); para o controle do manejo da irrigação (GONÇALVES et al., 2012; SILVA et al., 2012).

No trabalho é apresentado o desenvolvimento de um conjunto de planilhas eletrônicas para o controle do manejo da irrigação.

\section{MATERIAL E MÉTODOS}

O conjunto de planilhas eletrônicas denominado "manejo irriga", foi desenvolvido através da linguagem de programação Visual Basic for Applications (VBA). O usuário tem as opções de realizar o manejo da irrigação com base em dados climáticos: evapotranspiração de referência (ET) estimada pelo método de Penman-Monteith FAO 56 com dados da estação convencional, automática e com dados mínimos (temperatura máxima e mínima do ar); $\mathrm{ET}_{\mathrm{o}}$ estimada pelo método de Hargreaves-Samani e pelo tanque Classe "A".

Manejo com base na umidade do solo: pelo método direto (umidade padrão) e indiretamente, pelo controle da tensão de água no solo com o emprego de tensiômetro.

Manejo integrado (dados de clima e solo): ET $_{\text {o }}$ estimada por Penman-Monteith FAO 56 com dados de estação convencional e automática; ET estimada pelo método de Hargreaves-Samani e pelo tanque Classe "A", e umidade do solo determinada indiretamente, pelo controle da tensão de água no solo com o emprego de tensiômetro.

Para avaliar as planilhas desenvolvidas, foram obtidas medidas diárias de evaporação pelo tanque Classe "A", coeficiente do tanque e coeficiente de cultivo $(\mathrm{Kc})$ do melão para as diferentes fases fenológicas (Quadro 1), obtidos do trabalho realizado por Mota (2010) no município

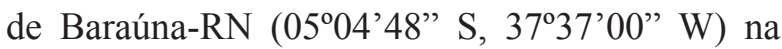
Chapada do Apodi. O solo da área experimental é um Cambissolo originado do calcário da Formação Jandaíra. De acordo com a classificação de Köppen o clima predominante na região é do tipo BSw'h', caracterizado por ser muito quente e semiárido, com a estação chuvosa se atrasando para o outono. O índice pluviométrico situa-se, em média, em torno dos $677 \mathrm{~mm} \mathrm{ano}^{-1}$.

\section{REVENG} 187-196p. 
Quadro 1. Coeficiente de cultivo $(\mathrm{Kc})$ nas diferentes fases fenológicas da cultura do melão

\begin{tabular}{ccc}
\hline Fases fenológicas & Kc & Período (dias) \\
\hline Inicial & 0,16 & 15 \\
Vegetativo & 0,33 & 18 \\
Frutificação & 1,08 & 18 \\
Maturação & 0,62 & 12 \\
\hline
\end{tabular}

Para as estimativas evapotranspiração de referência (ETo ) pelos métodos de Penman-Monteith FAO 56 com dados completos, Penman-Monteith FAO 56 com dados mínimos (temperatura máxima e mínima do ar) e Hargreaves-Samani (1985) disponíveis nas planilhas, foram obtidos dados diários da estação meteorológica convencional de Mossoró-RN, a saber: temperatura máxima e mínima do ar, umidade relativa do ar, velocidade do vento e número de horas de insolação, compreendendo o período de dezembro de 2005 a março de 2006.

A evapotranspiração de referência foi estimativa pelo método de Penman-Monteith, parametrizado pela FAO no seu manual número 56 (ALLEN et al., 1998), Equação 1.

$\mathrm{ET}_{0}=\frac{0,408 \ddot{\mathrm{A}}\left(\mathrm{R}_{\mathrm{n}}-\mathrm{G}\right)+\tilde{\mathrm{a}} \frac{900}{\mathrm{~T}_{\mathrm{m}}+273} \mathrm{u}_{2}\left(\mathrm{e}_{\mathrm{s}}-\mathrm{e}_{\mathrm{a}}\right)}{\ddot{\mathrm{A}}+\tilde{\mathrm{a}}\left(1+0,34 \cdot \mathrm{u}_{2}\right)}$

em que

$\mathrm{ET}_{\mathrm{o}}=$ evapotranspiração de referência, $\mathrm{mm} \mathrm{dia}^{-1}$;

$\mathrm{R}_{\mathrm{n}}=$ radiação líquida total do gramado, $\mathrm{MJ} \mathrm{m}^{-2} \mathrm{dia}^{-1}$; $\mathrm{G}=$ densidade do fluxo de calor no solo, $\mathrm{MJ} \mathrm{m}^{-2} \mathrm{dia}^{-1}$; $\mathrm{T}_{\mathrm{m}}=$ temperatura média diária do $\mathrm{ar},{ }^{\circ} \mathrm{C}$;

$\mathrm{u}_{2}=$ velocidade do vento média diária a $2 \mathrm{~m}$ de altura, $\mathrm{m} \mathrm{s}^{-1}$;

$\mathrm{e}_{\mathrm{s}}=$ pressão de saturação de vapor, $\mathrm{kPa}$;

$\mathrm{e}_{\mathrm{a}}^{\mathrm{s}}=$ pressão parcial de vapor, $\mathrm{kPa}$;

$\mathrm{e}_{\mathrm{s}}-\mathrm{e}_{\mathrm{a}}=$ déficit de saturação de vapor, $\mathrm{kPa}$;

$\Delta=$ declividade da curva de pressão de vapor no ponto de $\mathrm{T}_{\mathrm{m}}, \mathrm{kPa}^{\circ} \mathrm{C}^{-1}$;

$\gamma=$ coeficiente psicrométrico, $\mathrm{kPa}^{\circ} \mathrm{C}^{-1}$.

A pressão de saturação de vapor $\left(\mathrm{e}_{\mathrm{s}}\right)$ foi calculada em função da pressão de saturação da temperatura máxima e mínima do ar, Equações 2, 3 e 4.

$$
\mathrm{e}_{\mathrm{s}}=\frac{\mathrm{e}^{\mathrm{o}}\left(\mathrm{T}_{\mathrm{x}}\right)+\mathrm{e}^{\mathrm{o}}\left(\mathrm{T}_{\mathrm{n}}\right)}{2}
$$

$$
e^{o}\left(T_{x}\right)=0,6108 \exp \left(\frac{17,27 \cdot T_{x}}{T_{x}+237,3}\right)
$$

$$
e^{o}\left(T_{n}\right)=0,6108 \exp \left(\frac{17,27 \cdot T_{n}}{T_{n}+237,3}\right)
$$

A pressão de vapor parcial $\left(\mathrm{e}_{\mathrm{a}}\right.$ ) foi calculada utilizando a umidade relativa média $\left(\mathrm{UR}_{\mathrm{m}}\right)$, Equação 5.

$$
e_{a}=\frac{e_{s}\left(T_{m}\right) \cdot U R_{m}}{100}
$$

A declividade de curva de pressão de vapor foi obtida através da Equação 6.

$$
\Delta=\frac{4098\left[0,6108 \exp \left(\frac{17,27 \mathrm{~T}_{\mathrm{m}}}{\mathrm{T}_{\mathrm{m}}+237,3}\right)\right]}{\left(\mathrm{T}_{\mathrm{m}}+237,3\right)^{2}}
$$

A constante psicrométrica foi estimada pelas Equações 7 e 8 .

$$
\begin{aligned}
& \gamma=\frac{\mathrm{c}_{\mathrm{p}} \cdot \mathrm{p}}{\varepsilon \cdot \lambda}=0,665 \times 10^{-3} \cdot \mathrm{p} \\
& P=101,3\left(\frac{293-0,0065 \cdot Z}{293}\right)^{5,26}
\end{aligned}
$$

em que

$\mathrm{C}_{\mathrm{p}}=$ calor específico a pressão constante, $1,013 \times 10^{-}$

${ }^{3}, \mathrm{MJ} \mathrm{kg}^{-1}{ }^{\circ} \mathrm{C}^{-1}$;

$\mathrm{P}=$ pressão atmosférica, $\mathrm{kPa}$;

$\varepsilon=$ coeficiente de peso molecular de vapor de água do ar seco, 0,622;

$\lambda=$ calor latente de vaporização, 2,45 $\mathrm{MJ} \mathrm{kg}^{-1}$;

$\mathrm{Z}$ - altitude do local, $\mathrm{m}$. 
Calculou-se a distancia relativa Terra-Sol (dr), declinação solar ( $\delta$ ), ângulo de radiação no momento do por do sol $\left(\omega_{\mathrm{s}}\right)$ e duração máxima de brilho solar em um dia $(\mathrm{N})$, pelas Equações 9, 10, 11 e 12.

$$
\begin{aligned}
& d r=1+0,033 \cos \left(\frac{2 \pi}{365} J\right) \\
& \delta=0,409 \operatorname{sen}\left(\frac{2 \pi}{365} \mathrm{~J}-1,35\right) \\
& \omega_{s}=\arccos (-\tan \varphi \cdot \tan \delta) \\
& N=\frac{24 \cdot \omega_{s}}{\pi}
\end{aligned}
$$

em que

$\mathrm{d}_{\mathrm{r}}=$ distancia relativa Terra-Sol;

$\mathrm{J}=$ dia Juliano;

$\delta=$ declinação solar, radianos;

$\varphi=$ latitude local, radianos;

$\omega_{\mathrm{s}}=$ ângulo de radiação no momento do por do sol, radianos;

$\mathrm{N}=$ duração máxima de brilho solar em um dia, h.

O cálculo da radiação no topo da atmosfera $\left(R_{a}\right)$ foi obtido pela equação 13 , em seguida calculou-se a radiação solar global $\left(R_{s}\right)$ e radiação solar em um dia sem nuvens $\left(\mathrm{R}_{\mathrm{so}}\right)$, Equações 14 e 15.

$$
\begin{aligned}
& R_{a}=\frac{24(60)}{\pi} G_{s c} \cdot d_{r}\left(\omega_{s} \cdot \operatorname{sen} \varphi \cdot \operatorname{sen} \delta+\cos \varphi \cdot \cos \delta \cdot \operatorname{sen} \omega_{s}\right) \\
& R_{s}=R_{a}\left(\mathrm{a}+\mathrm{b} \frac{\mathrm{n}}{\mathrm{N}}\right) \\
& R_{s o}=R_{a}(\mathrm{a}+\mathrm{b})
\end{aligned}
$$

em que

$\mathrm{R}_{\mathrm{a}}=$ radiação no topo da atmosfera, $\mathrm{MJ} \mathrm{m} \mathrm{m}^{-2} \mathrm{dia}^{-1}$;

$\mathrm{G}_{s c}=$ constante, $0,0820 \mathrm{MJ} \mathrm{m}^{-2} \mathrm{dia}^{-1}$;

$\mathrm{a}$ e $\mathrm{b}=$ coeficientes locais, $\mathrm{a}=0,25$ e $\mathrm{b}=0,50$;

$\mathrm{n}=$ número de horas de brilho solar do dia, $\mathrm{h}$;

$\mathrm{R}_{\mathrm{s}}=$ radiação solar global, $\mathrm{MJ} \mathrm{m} \mathrm{m}^{-2} \mathrm{dia}^{-1}$;

$\mathrm{R}_{\mathrm{so}}=$ radiação solar em um dia sem nuvens, MJ $\mathrm{m}^{-2} \mathrm{dia}^{-1}$.

O saldo de radiação de onda curta $\left(\mathrm{R}_{\mathrm{ns}}\right)$ é obtido pela equação 16 e o saldo de radiação de onda longa $\left(\mathrm{R}_{\mathrm{nl}}\right)$, pela Equação 17.

$$
\begin{aligned}
& R_{n s}=(1-\alpha) \cdot R_{s} \\
& \mathrm{R}_{\mathrm{nl}}=\sigma\left(\frac{\mathrm{T}_{\mathrm{x}, \mathrm{k}^{4}}+\mathrm{T}_{\mathrm{n}, \mathrm{k}^{4}}}{2}\right)\left(0,34-0,14 \sqrt{\mathrm{e}_{\mathrm{a}}}\right)\left(1,35 \frac{\mathrm{R}_{\mathrm{s}}}{\mathrm{R}_{\mathrm{so}}}-0,35\right)
\end{aligned}
$$

em que

$\alpha=$ albedo da superfície (grama), 0,23;

$\sigma=$ constante de Stefan-Boltzmann, 4,903 x $10^{-9}$ $\mathrm{MJ} \mathrm{m}^{-2} \mathrm{dia}^{-1}$;

$\mathrm{T}_{\mathrm{x}, \mathrm{k}}=$ temperatura máxima observada durante o período de 24 horas, $\mathrm{K}$;

$\mathrm{T}_{\mathrm{n}, \mathrm{k}}=$ temperatura mínima observada durante o período de 24 horas, $\mathrm{K}$.

O saldo de radiação $\left(R_{n}\right)$ é a diferença entre o saldo de radiação de onda curta $\left(\mathrm{R}_{\mathrm{ns}}\right)$ e o saldo de radiação de onda longa $\left(\mathrm{R}_{\mathrm{nl}}\right)$, Equação 18 .

$$
R_{n}=R_{n s}-R_{n l}
$$

O fluxo de calor no solo (G) é considerado igual à zero, pois segundo Allen et al. (1998), a magnitude de fluxo de calor no solo para períodos diários ou dez dias sob uma superfície de referência é relativamente pequena, podendo ser ignorado.

$\mathrm{Na}$ estimativa da evapotranspiração de referência pela equação de Penman-Monteith FAO 56 com dados mínimos (temperatura máxima e mínima do ar), a pressão parcial de vapor $\left(\mathrm{e}_{\mathrm{a}}\right)$ foi calculada substituindo a temperatura do ponto

\section{REVENG 187-196p.}


de orvalho pela temperatura mínima diária do ar menos $2{ }^{\circ} \mathrm{C}\left(\mathrm{T}_{\mathrm{n}}-2^{\circ} \mathrm{C}\right)$, conforme sugerido por Allen et al. (1998) para climas semiáridos, Equação 19.

$$
e_{a}=0,6108 \exp \left(\frac{17,27 T_{d}}{T_{d}+237,3}\right)
$$

em que

$\mathrm{e}_{\mathrm{a}}=$ pressão parcial de vapor, $\mathrm{kPa}$;

$\mathrm{T}_{\mathrm{d}}=$ temperatura do ponto de orvalho, ${ }^{\circ} \mathrm{C}$.

A radiação solar em um dia sem nuvens $\left(\mathrm{R}_{\mathrm{so}}\right)$ foi calculada através da Equação 20.

$$
R_{s o}=\left(0,75+2 \times 10^{-5} \cdot Z\right) \cdot R_{a}
$$

$\mathrm{Na}$ estimativa da radiação solar global foi utilizado o método de Hargreaves-Samani (1982), Equação 21.

$$
R_{s}=K_{r s} \cdot\left(T_{x}-T_{n}\right)^{0,5} \cdot R_{a}
$$

em que

$\mathrm{R}_{\mathrm{a}}=$ radiação no topo da atmosfera, $\mathrm{MJ} \mathrm{m}^{-2} \mathrm{dia}^{-1}$; $\mathrm{K}_{\mathrm{rs}}^{\mathrm{a}}=$ coeficiente empírico igual a 0,16 na região continental e 0,19 na região costeira;

$\mathrm{T}_{\mathrm{x}}=$ temperatura máxima do $\mathrm{ar},{ }^{\circ} \mathrm{C}$;

$\mathrm{T}_{\mathrm{n}}^{\mathrm{x}}=$ temperatura mínima do $\mathrm{ar},{ }^{\circ} \mathrm{C}$.

A estimativa de ET ${ }_{\mathrm{o}}$ pelo método de HargreavesSamani (1985) é obtida utilizando-se a Equação 22.

$$
E T_{o}=0,0023 \cdot\left(T_{m}+17,8\right) \cdot\left(T_{x}-T_{n}\right)^{0,5} \cdot R_{a} \cdot 0,408
$$

em que

$\mathrm{ET}_{\mathrm{o}}=$ evapotranspiração de referência, $\mathrm{mm}$ dia $^{-1}$. As demais variáveis estão citadas acima.

A estimativa de $\mathrm{ET}_{\mathrm{0}}$ pelo tanque Classe "A" é obtida utilizando-se a Equação 23.

$$
E T_{o}=K p \cdot E C A
$$

em que

$\mathrm{ET}_{\mathrm{o}}=$ evapotranspiração de referência pelo tanque Classe "A", mm dia ${ }^{-1}$;

$\mathrm{Kp}=$ coeficiente do tanque; adimensional;

$\mathrm{ECA}=$ evaporação do tanque Classe " $\mathrm{A}$ ", $\mathrm{mm} \mathrm{dia}{ }^{-1}$.

A evapotranspiração da cultura (ETc) é obtida pela Equação 24.

$$
E T c=E T_{o} \cdot K c
$$

em que

$\mathrm{ETc}=$ evapotranspiração da cultura, $\mathrm{mm} \mathrm{dia}{ }^{-1}$; $\mathrm{ET}_{\mathrm{o}}=$ evapotranspiração de referência obtida pelo respectivo método, $\mathrm{mm} \mathrm{dia}^{-1}$;

$\mathrm{Kc}=$ coeficiente de cultivo, adimensional.

\section{RESULTADOS E DISCUSSÃO}

O conjunto de planilhas eletrônicas desenvolvidas parte de uma tela inicial (Figura 1) e ao clicar no botão "DADOS GERAIS", o usuário terá acesso a uma nova tela para o preenchimento dos dados gerais (Figura 2). Os campos serão preenchidos conforme o tipo de manejo adotado.

A demanda hídrica da cultura do melão (ETc) com a estimativa da evapotranspiração de referência obtida pelo tanque Classe " $A$ " na fase inicial foi $17,22 \mathrm{~mm}$, no período vegetativo de 43,68 mm, na fase de maior demanda hídrica (frutificação) foi encontrado valor 128,06 mm e na última fase (maturação), a demanda hídrica foi $36,83 \mathrm{~mm}$. A evapotranspiração da cultura (ETc) durante todo o seu ciclo foi $225,79 \mathrm{~mm}$, com valores diários de 1,$15 ; 2,43 ; 7,11$ e $3,07 \mathrm{~mm}$ para os períodos inicial, vegetativo, frutificação e maturação, respectivamente (Figura 3).

Os valores encontrados por Mota (2010) foram 16,$75 ; 43,91 ; 127,65$ e $37,00 \mathrm{~mm}$ para os períodos inicial, vegetativo, de frutificação e de maturação, respectivamente, totalizando $225,32 \mathrm{~mm}$ em todo o ciclo. Com ETc diária de 1,12; 2,44; 7,09 e 3,08 $\mathrm{mm}$, respectivamente para os mesmos períodos citados acima.

Com base nos resultados obtidos com o uso das planilhas "manejo irriga" testando os dados obtidos por Mota (2010), verificou-se que ambos 


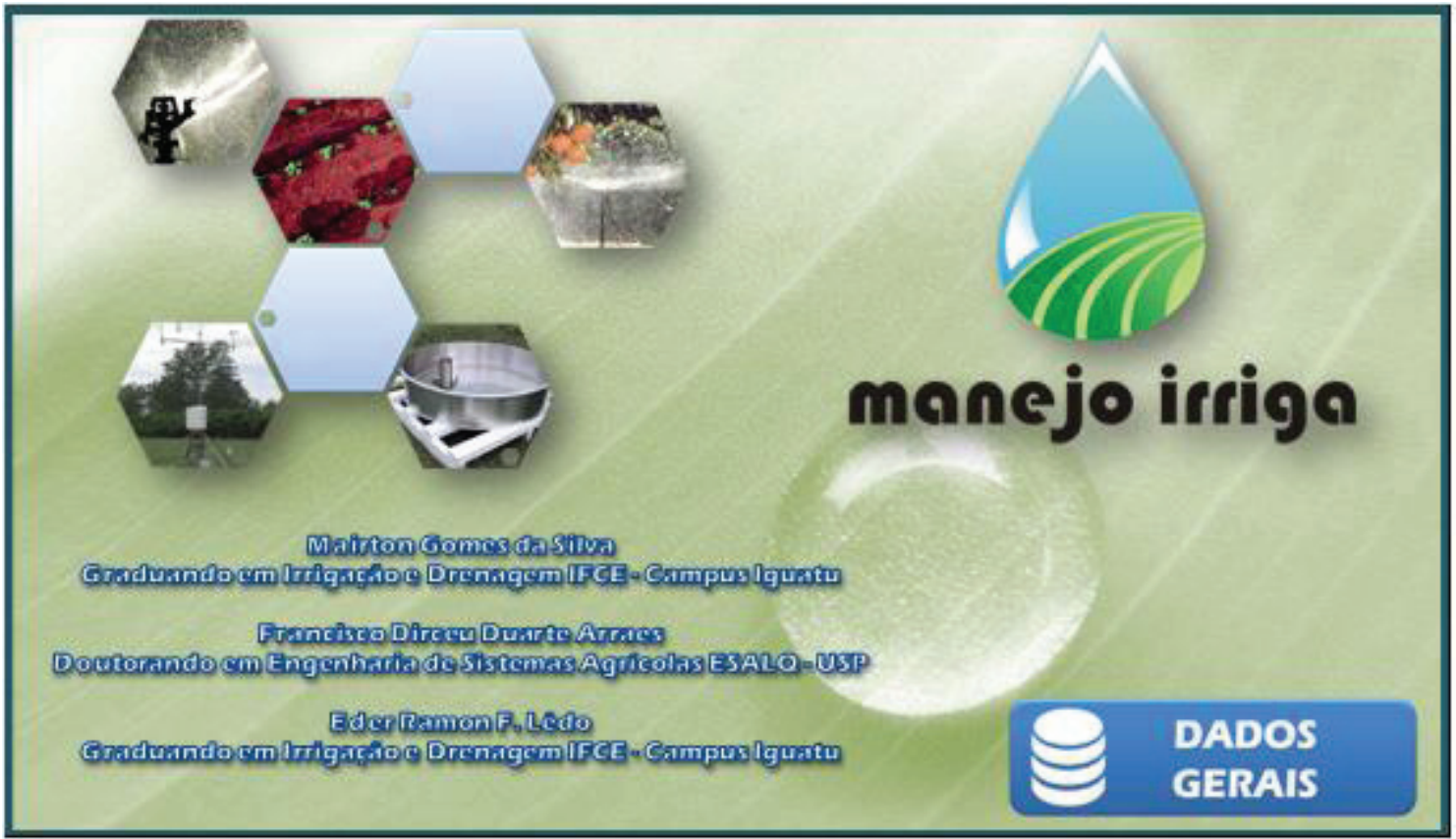

Figura 1. Tela inicial do conjunto de planilhas "manejo irriga".

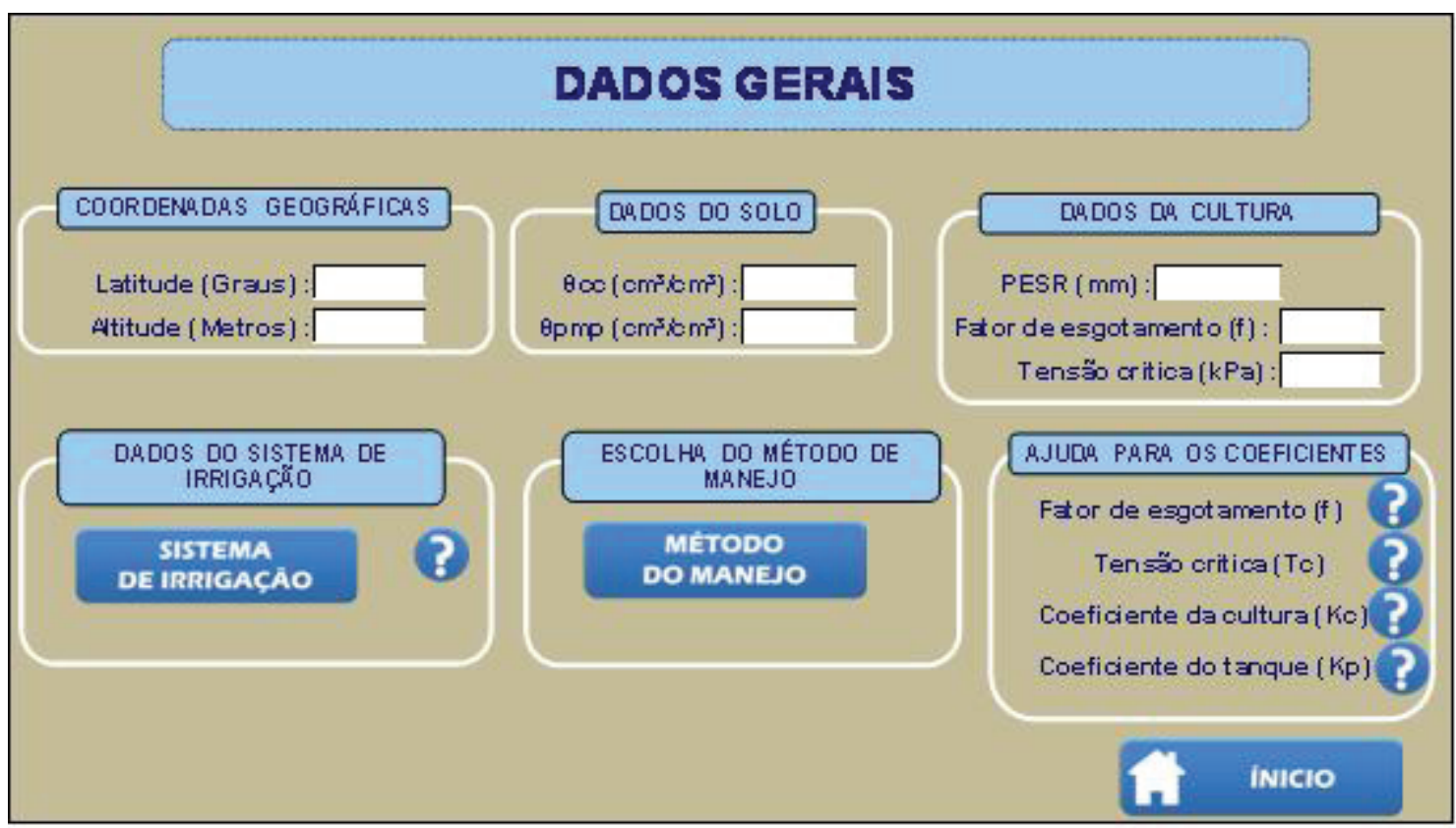

Figura 2. Tela dos dados gerais.

os resultados foram próximos, com valores de ETc durante todo o ciclo determinada por Mota (2010) de $225,32 \mathrm{~mm}$ e encontrado pelo "manejo irriga" de $225,79 \mathrm{~mm}$. O conjunto de planilhas "manejo irriga" mostrou-se prático e rápido na execução dos cálculos envolvidos na determinação das necessidades hídricas das culturas.
A demanda hídrica da cultura do melão com $\mathrm{ET}_{\mathrm{o}}$ estimada por Penman-Monteith FAO 56 (PM-FAO 56) com dados completos foi de $17,27 \mathrm{~mm}(1,15$ $\mathrm{mm}$ dia $\left.^{-1}\right)$ no período inicial, $43,10 \mathrm{~mm}(2,39 \mathrm{~mm}$ $\left.\operatorname{dia}^{-1}\right)$ no período vegetativo, $120,33 \mathrm{~mm}(6,68 \mathrm{~mm}$ $\left.\operatorname{dia}^{-1}\right)$ na fase de frutificação e de $37,28 \mathrm{~mm}(3,11$ $\mathrm{mm}$ dia $^{-1}$ ) na fase de maturação, com demanda

\section{REVENG}

187-196p. ENGENHARIA NA AGRICULTURA, VIÇOSA - MG, V.21 N.2, MARÇO / ABRIL 2013 


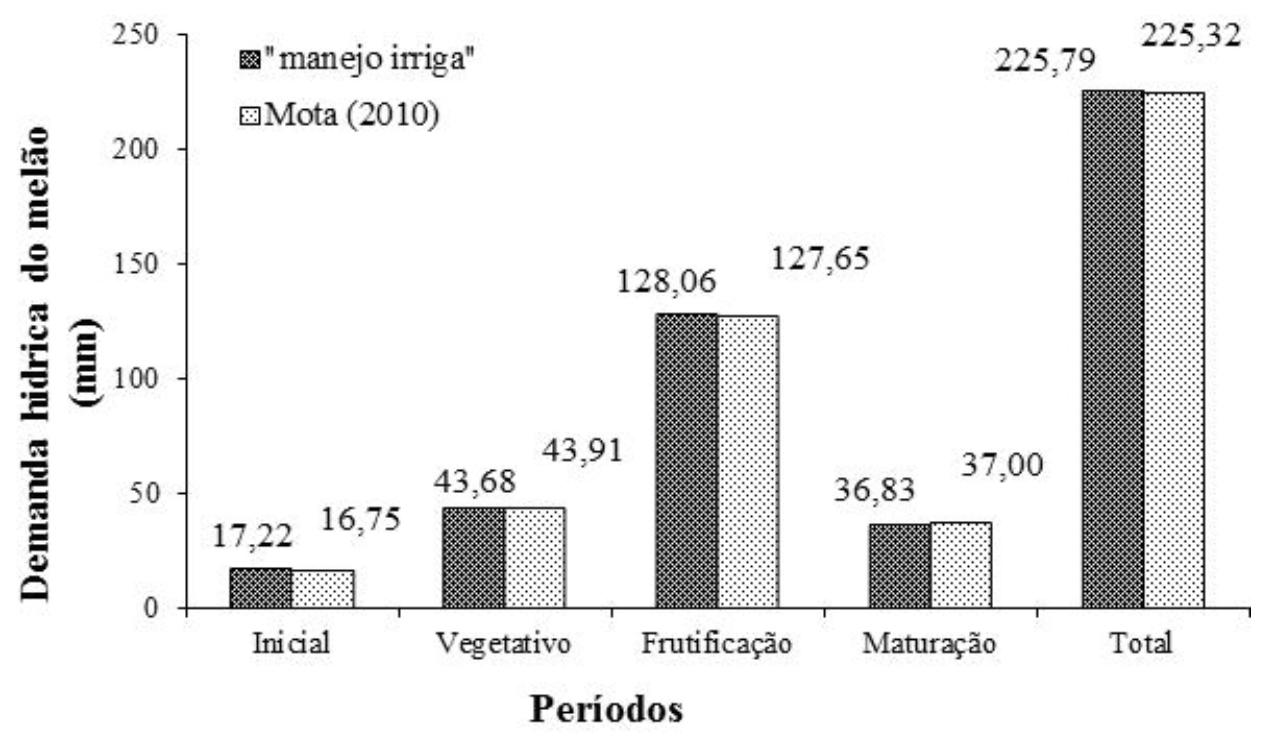

Figura 3. Demanda hídrica do meloeiro (ETc) determinada por Mota (2010) e com o emprego das planilhas "manejo irriga" com ET obtida pelo tanque Classe "A".



Figura 4. Demanda hídrica do meloeiro (ETc) determinada por Mota (2010) e com o emprego das planilhas eletrônicas "manejo irriga" com ET estimada por Penman-Monteith FAO 56 com dados completos e dados mínimos (Tx e Tn).

hídrica durante todo ciclo da cultura de 217,97 $\mathrm{mm}$. Em relação aos valores encontrados por Mota (2010) com uso do tanque Classe "A", na fase de frutificação foi encontrado a maior diferença entre os dois métodos, sendo que o método de PM-FAO 56 subestima em 7,32 mm (5,73\%). Durante todo ciclo da cultura PM-FAO 56 subestimada em 7,35 $\mathrm{mm}(3,26 \%)$ em relação aos valores obtidos com o emprego do tanque Classe "A" (Figura 4).
A demanda total da cultura (ETc) com estimativas de ET por PM-FAO 56 com dados de temperatura máxima e mínima do ar, foi menor em $7,69 \%(17,33 \mathrm{~mm})$ dos valores encontrados por Mota (2010) com estimativas de $\mathrm{ET}_{0}$ pelo tanque Classe "A". Os valores foram de 15,99 mm (1,07 $\left.\mathrm{mm} \mathrm{dia}^{-1}\right), 40,33 \mathrm{~mm}\left(2,24 \mathrm{~mm} \mathrm{dia}{ }^{-1}\right), 111,94$ $\mathrm{mm}\left(6,22 \mathrm{~mm} \mathrm{dia}^{-1}\right)$ e $39,74 \mathrm{~mm}\left(3,31 \mathrm{~mm} \mathrm{dia}^{-1}\right)$ para o período inicial, vegetativo, frutificação e maturação, respectivamente e demanda durante 


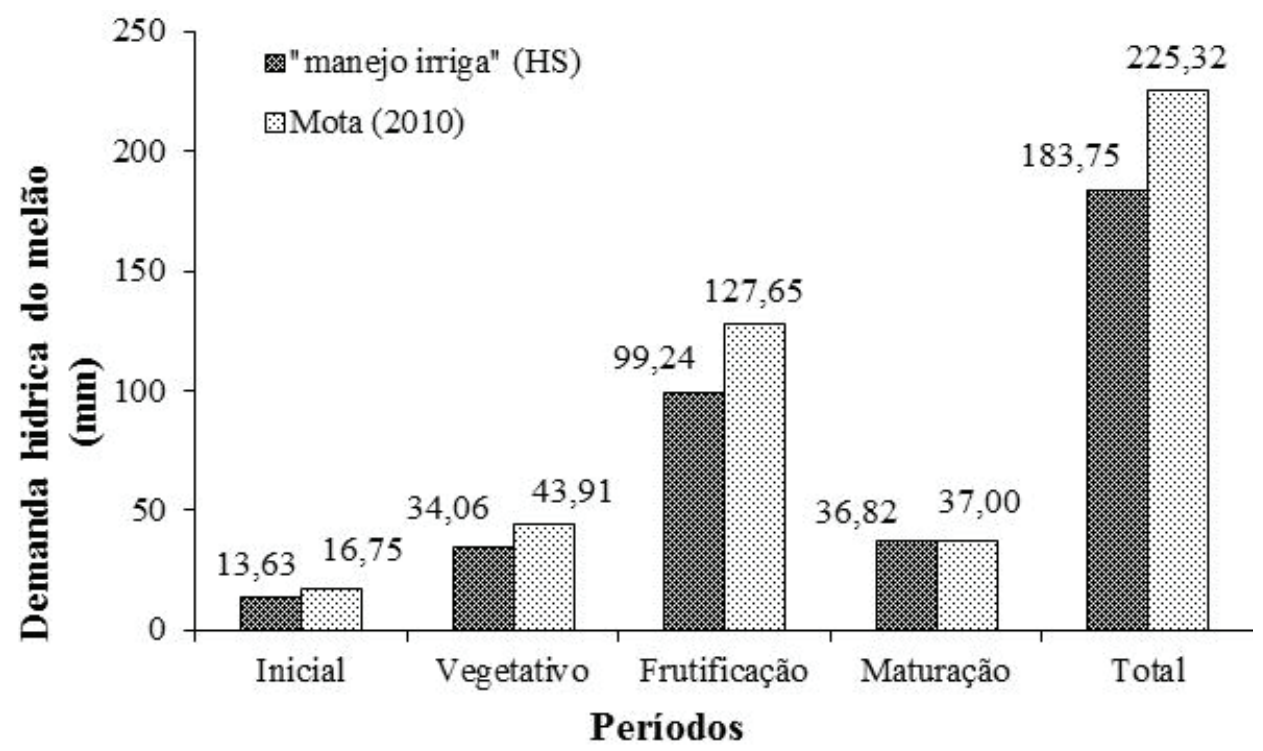

Figura 5. Demanda hídrica do meloeiro (ETc) determinada por Mota (2010) e com o emprego das planilhas eletrônicas "manejo irriga" com ET estimada pelo método de Hargreaves-Samani (1985).

todo o ciclo de $208,00 \mathrm{~mm}$.

A estimativa das necessidades hídricas das culturas com $\mathrm{ET}_{\mathrm{o}}$ obtida por PM-FAO 56 com dados de temperatura máxima e mínima do ar é uma possibilidade de ser utilizado com apenas essas duas variáveis no aplicativo. Sendo que no "manejo irriga", utiliza-se a velocidade do vento fixa de $2 \mathrm{~m} \mathrm{~s}^{-1}$ recomendada pela FAO (ALLEN et al., 1998) quando não se dispõe de dados de velocidade do vento, sendo recomendo o ajuste de uma velocidade fixa que melhor represente as condições climáticas da região do manejo.

Trabalhos foram realizados para avaliar a $\mathrm{ET}_{\text {。 }}$ com dados mínimos e utilizaram-se o valor fixo de velocidade do vento de $2 \mathrm{~m} \mathrm{~s}^{-1}$ recomendada pela FAO (ALLEN et al., 1998), no sul da Bulgária (POPOVA et al., 2006); na Tunísia (JABLOUN; SAHLI, 2008), e verificaram que esse valor tende a superestimar $\mathrm{ET}_{\mathrm{o}}$ padrão. Porém, no sul de Ontário no Canadá (SENTELHAS et al., 2010), os autores concluíram que esse procedimento é aceitável para estimar $\mathrm{ET}_{\mathrm{o}}$ diária.

As necessidades hídricas do meloeiro com ET $_{\mathrm{o}}$ estimada pelo método de Hargreaves-Samani (1985) foram de 13,63 $\mathrm{mm}$ no período inicial, $34,06 \mathrm{~mm}$ no período vegetativo, 99,24 $\mathrm{mm}$ para o período de frutificação e $36,82 \mathrm{~mm}$ no período de maturação, com demanda total de $183,75 \mathrm{~mm}$ (Figura 5). Todas as fases subestimam os valores encontrados por Mota (2010) com ET obtida pelo tanque Classe "A", menos a fase de maturação que foi encontrado a mesma demanda hídrica, a fase inicial subestima em 3,12 mm (18,63\%), fase vegetativa em $9,85 \mathrm{~mm}(22,43 \%)$ e na de frutificação com maior subestimativa, de 28,41 $\mathrm{mm}(22,26 \%)$.

Durante todo ciclo da cultura a subestimativa da demanda hídrica do meloeiro com o emprego das planilhas "manejo irriga" em comparação aos valores encontrados por Mota (2010), foram da ordem de $41,57 \mathrm{~mm}(18,45 \%)$.

\section{CONCLUSÕES}

- O conjunto de planilhas "manejo irriga" mostrou-se prático e rápido na execução dos cálculos envolvidos na determinação das necessidades hídricas da cultura;

- A simulação da demanda hídrica do melão com estimativas de $\mathrm{ET}_{\mathrm{o}}$ pelo tanque Classe "A" obtida através do conjunto de planilhas "manejo irriga", mostrou-se valores próximos aos obtidos no experimento;

- A utilização de métodos de estimativas de $\mathrm{ET}_{\text {o }}$ pode ser decisivos no estudo das necessidades hídricas das culturas e manejo de irrigação, contribuindo sobremaneira para o uso racional da água na agricultura irrigada.

\section{REVENG}




\section{REFERÊNCIAS BIBLIOGRÁFICAS}

ALBUQUERQUE, P.E.P.; ANDRADE, C.L.T. Planilha eletrônica para a programação da irrigação de culturas anuais. Sete Lagoas, MG. Embrapa Milho e Sorgo, 2001. 14p. (Circular Técnica, 10).

ALLEN, R.G.; PEREIRA, L.; RAES, D.; SMITH, $M$. Crop evapotranspiration: guidelines for computing crop water requirements. Rome: FAO, 1998. (Irrigation and Drainage Paper, 56).

BARRETO, A.N.; FACIOLI, G.G.; SILVA, A.A. Operação e o manje dos sistemas de irrigação. In: BARRETO, A. N.; SILVA, A. A. G.; BOLFE, E. L. Irrigação e drenagem na empresa agrícola: impacto ambiental $\mathbf{x}$ sustentabilidade. Aracaju: Embrapa Tabuleiros Costeiros, 2004. cap.5, p.173-204.

BUSATO, C.C.M.;BUSATO, C. Evapotranspiração da videira em Santa Teresa, ES: coeficiente de cultura "único" padrão boletim FAO 56. Revista Verde de Agroecologia e Desenvolvimento Sustentável, Mossoró-RN, v.6, n.4, p.251-257, out/dez. 2011.

CONCEIÇÃO, M.A.F. Planilha eletrônica para manejo da irrigação em pequenas propriedades. In: Congresso Nacional de Irrigação e Drenagem, 20, Uberaba - MG. Anais... Uberaba - MG: ABID, 2010. CD Rom.

GONÇALVES, F.M.; TIMBÓ, Á.P.; COSTA, R.N.T.; MENEZES, J.W.M.; CANAFÍSTULA, F.J.F.; MACEDO, A.B.M. Manejo móvel: Uma ferramenta facilitadora no manejo da irrigação localizada por gotejamento. In: I Inovagri International Meeting \& IV Workshop Internacional de Inovações Tecnológicas na Irrigação, 2012, Fortaleza, CE. Anais... Fortaleza, CE, 2012

HARGREAVES, G.H.; SAMANI, Z.A. Estimating potential evapotranspiration. Journal of Irrigation and Drainage Engineering, New York, v.108, p.225-230, 1982.

HARGREAVES, G.H.; SAMANI, Z.A. Reference crop evapotranspiration from temperature. Applied Engineering Agricuture, v.1, n.2, p.96-99, 1985.

JABLOUN, M.; SAHLI, A. Evaluation of
FAO-56 methodology for estimating reference evapotranspiration using limited climatic data application to Tunisia. Agricultural Water Management, New York, v.95, n.06, p.707-715, 2008.

MIRANDA, F.R. Irrigacaju: Planilha Eletrônica para o Manejo da Irrigação na Produção Integrada de Caju. Fortaleza, CE: Embrapa Agroindústria Tropical, 2005. 8p. (Circular Técnica, 23).

MOTA, J.C.A. Componentes do balanço de água em um Cambissolo cultivado com meloeiro irrigado por gotejamento, com e sem cobertura da superfície. 2010. 106p. Tese (Doutorado em Solos e Nutrição de Plantas) - Escola Superior de Agricultura "Luiz de Queiroz", Piracicaba, 2010.

NASCIMENTO, P.S.; BASSOI, L.H.; PAZ, V.P.S. Planilha eletrônica para auxilio à tomada de decisão em manejo de irrigação. Irriga, Botucatu-SP, v.17, n.1, p.1-15, jan/mar. 2012.

OLIVEIRA, E.M.; OLIVEIRA, R.A.; SEDIYAMA, G.C.; CECON, P.R.; DRUMOND, L.C.D. Análise do coeficiente e o desempenho do irrigâmetro e a influência dos elementos do clima na estimativa da evapotranspiração. Revista Engenharia na Agricultura, Viçosa-MG, v.19, n.4, p.348-360, julho/agosto, 2011.

OLIVEIRA, G.M.; LEITÃO, M.M.V.B.R.; ALMEIDA, A.C. Determinação da evapotranspiração e dos coeficientes de cultura para as diferentes fases de desenvolvimento do melão (Cucumis melo L.) na região Norte da Bahia. Revista Verde de Agroecologia e Desenvolvimento Sustentável, Mossoró-RN, v.5, n.2, p.142-151, abr/jun. 2010.

PAULA, J.A.A.; MEDEIROS, J.F.; SOBRINHO, J.E.; CARDOSO, E.A.; MENDONÇA, V. Sistema informatizado para o manejo da irrigação na cultura da mangueira CV. 'Tomy Atkins' para a Chapada do Apodi. Revista Agropecuária Científica no SemiÁrido, Patos-PB, v.7, n.4, p.01-04, out/dez. 2011.

PINTO, F.A.; FREITAS, C.A.S.; RIBEIRO, R.S.F. Planilha eletrônica para planejamento de calendários de irrigação. In: I Workshop Internacional de Inovações Tecnológicas na Irrigação \& I Conferência sobre Recursos hídricos do Semiárido Brasileiro, 2007, Sobral - CE. Anais... Sobral - CE: Instituto INOVAGRI, 2007. CD Rom. 
POPOVA, Z.; KERCHEVA, M.; PEREIRA, L.S. Validation of the FAO methodology for computing $\mathrm{ET}_{\text {o }}$ with limited data. Application to South Bulgaria. Journal of Irrigation and Drainage, New York, v.55, n.2, p.201-215, 2006.

SILVA, I.N.; OLIVEIRA, J.B.; FONTES, L.O.; BRASIL, P.P. SILVA, M.G. Estimativa da evapotranspiração de referência com dados mínimos para o Cariri oeste cearense, Revista Agropecuária Científica no Semi-Árido, PatosPB, v.6, n.3, p.42-48, jul/set. 2010.

SILVA, M.G.; ARRAES, F.D.D.; LEDO, E.R.F.; NOGUEIRA, D.H. Desenvolvimento de um aplicativo computacional para o controle do manejo da irrigação. In: I Inovagri International Meeting
\& IV Workshop Internacional de Inovações Tecnológicas na Irrigação, 2012, Fortaleza, CE. Anais... Fortaleza, CE, 2012.

SILVA, V.P.R.; FILHO, A.F.B.; SILVA, B.B.; CAMPOS, J.H.B.C. Desenvolvimento de um sistema de estimativa da evapotranspiração de referência, Revista Brasileira de Engenharia Agrícola e Ambiental, Campina Grande-PB, v.9, n. 4 , p.547-553, 2005.

SENTELHAS, P.C.; GILLESPIE, T.J.; SANTOS, E. A. Evaluation of FAO Penman-Monteith and alternative methods for estimating reference evapotranspiration with missing data in Southern Ontario, Canada. Agricultural Water Management, New York, v.97, n.5, p.635-644, 2010. 